\title{
DISTRIBUCION, BATIMETRIA Y ALIMENTACION DE EPIGONUS CRASSICAUDUS DE BUEN, 1959 (PERCIFORMES: EPIGONIDAE) EN LA COSTA DE CHILE
}

\section{DISTRIBUTION, BATHYMETRY AND FOOD OF EPIGONUS CRASSICAUDUS DE BUEN, 1959 (PERCIFORMES:EPIGONIDAE) IN THE COAST OF CHILE}

\author{
Elson Leal ${ }^{1}$, Francisco Contreras $^{1} \&$ Ciro Oyarzún ${ }^{2}$ \\ ${ }^{1}$ Departamento de Evaluación de Recursos. División de Investigación Pesquera. Instituto de Fomento Pesquero \\ (IFOP).Valparaíso, Chile. elson.leal@ifop.cl, \\ ${ }^{2}$ Departamento de Oceanografía, Universidad de Concepción, Chile
}

\begin{abstract}
RESUMEN
Se estudió la distribución batimétrica y latitudinal de besugo (Epigonus crassicaudus) en la costa de Chile analizando los registros de la pesquería y a través de información complementaria recopilada desde los lances de pesca realizados en la evaluación directa de merluza común. Adicionalmente, se reportan antecedentes sobre los hábitos alimenticios de besugo a través de análisis del contenido estomacal de ejemplares recolectados en la zona central de Chile. Según la información de la actividad comercial, el recurso concentraría su distribución entre Valparaíso (3304'S) y Puerto Montt (41 46'S). Sin embargo, el crucero de evaluación, registra capturas positivas de besugo desde su limite norte de operación $\left(29^{\circ} 00^{\prime} S\right)$. En relación a la batimetría, tanto la frecuencia de lances positivos como la captura, mostraron un patrón coincidente en ambas fuentes de información, evidenciando una concentración del recurso en el rango de profundidad de 300 a $400 \mathrm{~m}$. Respecto de la alimentación, las presas más consumidas por E. crassicaudus fueron organismos planctónicos representados por eufáusidos y estomatópodos. Apareciendo ocasionalmente algunos componentes del bentos en su dieta. Los resultados del presente estudio sugieren que E. crassicaudus presentaría hábitos migratorios desde el pélagos al bentos, determinado por la oferta ambiental de alimento, concentrando su distribución sobre la plataforma y talud continental. Además se confirmó una distribución latitudinal más amplia del recurso de la reconocida a través de información exclusiva de la pesquería.
\end{abstract}

Palabras Claves: alimentación, batimetría, besugo, distribución, Chile.

\begin{abstract}
By mans of the fisheries activity records and the information of tralws sampling carried out in surveys of common hake the batimetric and latitudinal distributions of cardinal fish (Epigonus crassicaudus) in the Chilean coast were studied. Aditionality, information on feeding habits of cardinal fish through analysis of the gastric content on samples collected in the central zone from Chile are reported. According to fishery data the cardinal fish would concentrate their distribution

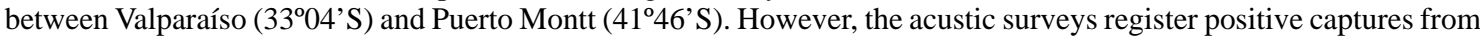
north limit of their operation $\left(29^{\circ} 00^{\prime} \mathrm{S}\right)$. Deep distribution showed coincidence from the two sources information so much in the throw positives frequency like in capture, indicating that the resource would concentrate their distributon in depth range of 300 to $400 \mathrm{~m}$. Regarding the feeding, although some bentics components were registered in their diet, the preys more consumed by E. crassicaudus were planctonics organisms represented by eufausids and stomatopods.

The result of present study suggest that E. crassicaudus would present migratory habits from the pelagics to the bentics environmental determined by the offer of food, concentrating their distribution on the talud and continental shelf. A wider latitudinal distribution acquaintance through the fisheries activity data was also confirmed.
\end{abstract}

KEYwORDs: Feeding, bathimetry, cardinal fish, distribution, Chile. 


\section{INTRODUCCION}

La pesquería de besugo (Epigonus crassicaudus De Buen 1959), partió en Chile formando parte de la fauna acompañante de merluza común y merluza del sur, registrándose por primera vez en el anuario estadístico de pesca en el año 1992 con 579 ton (Sernapesca 1992). A partir del año 1998 se transforma en pesca objetivo de la flota arrastrera industrial de Chile centro-sur. La mayor captura, se registró el año 2000, con cerca de 6 mil ton. A partir del año 2003 el desembarque ha fluctuado en torno a las 2 mil ton que corresponde a la cuota de captura establecida anualmente.

El genero Epigonus es uno de los cinco pertenecientes a la familia Epigonidae, cuya distribución es del tipo cosmopolita asociada a pendientes continentales, insulares y montes submarinos (Jonson 1984). Catalogados por Parín (1987) como peces mesobentico-pelágico relacionados principalmente al fondo. Algunas especies como E. telescopus, E. denticulatus, E. macrops se encuentran ampliamente distribuidas y otras como en el caso de besugo presentan un restringido rango de distribución (Abramov 1992). La información disponible en Chile sobre batimetría y distribución de E. crassicaudus se basa en los registros obtenidos desde la pesquería, la cual indica que esta especie se encontraría presente entre Valparaíso $\left(33^{\circ} 30^{\prime} S\right)$ y Puerto Montt $\left(42^{\circ} \mathrm{S}\right)$ a profundidades entre 100 y $500 \mathrm{~m}$ (Wiff et al. 2008). Respecto de su biología los antecedentes formales disponibles son escasos. Arancibia \& Melendez (1984) describen la dieta de besugo en la zona comprendida entre la Bahía Chanco y el Golfo de Arauco, indicando que E crassicuadus presenta hábitos alimenticios preferentemente pelágico y con aporte de pequeños peces demersales y de organismos epibéntónicos. Galvez et al. (2002) reportan antecedentes sobre los parámetros de la relación longitud-peso y estructura de longitudes del recurso en la pesquería.

El objetivo del presente estudio fue establecer, a partir de información obtenida desde las bitácoras de pesca, la distribución latitudinal y batimétrica de E. crassicaudus en la costa de Chile y contrastar los resultados a través del análisis de información complementaria e independiente, recopilada desde los lances de pesca realizados en los cruceros de evaluación hidroacústica de merluza común. Adicionalmente, se estudian los hábitos alimenticios de besugo a través de análisis del contenido estomacal de ejemplares recolectados en la costa central de Chile.

\section{MATERIALES Y METODOS}

La distribución batimétrica y latitudinal de $E$. crassicaudus en la costa de Chile, se estudió a partir de dos fuentes de información.

Se examinó la información recopilada del monitoreo de la pesquería desarrollada por el programa de seguimientos de recursos de aguas profundas del Instituto de Fomento Pesquero (IFOP). El programa contempla 2 a 3 embarques mensuales en viajes de pesca destinados a besugo. Se analizaron 10 años de información en el periodo 1997-2006.

Además se analizó la información proveniente de los cruceros de evaluación directa de merluza común, en donde se registra besugo como fauna acompañante en los lances de pesca realizados en la prospección (Lillo et al. 2005). Se analizaron nueve años de información. Cada dos años en el periodo 1993-1999 y cada año desde el 2000 al 2005 (excepto el 2003). Los cruceros de evaluación directa de merluza se realizan en el área comprendida entre los $29^{\circ}$ y $41^{\circ}$ de latitud sur y desde la primera milla de la costa hasta el veril de los $500 \mathrm{~m}$ de profundidad.

Tanto en el monitoreo de la pesquería como en los cruceros de evaluación directa, se registra información referente a la profundidad, posición, captura, esfuerzo y hora del lance, entre otras variables. Por lo tanto en el presente estudio se realizó un análisis comparativo entre ambas fuentes de información, incluyendo distribución de frecuencia de lances y capturas del recurso en relación a la latitud y profundidad.

Para el estudio de alimentación se analizaron los estómagos de 171 especimenes de E. crassicaudus, obtenidos como fauna acompañante en la pesquería de arrastre del camarón (Pleuroncodes monodon) realizada en el Golfo de Arauco (36 $6^{\circ}-37^{\prime}$ S) en los meses de Enero, Febrero y Abril de 1993.

Luego de identificar el sexo, medir y pesar cada ejemplar, su estómago fue extraído y preservado en formalina al 10\%. Posteriormente, la identificación de las presas se realizó en laboratorio consultando bibliografía especializada. El éxito de la identificación de especies de presas dependió del grado de digestión que presentó el contenido estomacal de cada 
ejemplar. Cuando la identificación de las presas no fue posible se optó por denominarlos restos no identificados (RNI).

En el análisis de los datos se utilizó el Indice de Importancia Relativa (IIR) (Pinkas et al.1971; Berg 1979) y con fines comparativos se usó el Índice de Similitud Porcentual (ISP) (Whittaker 1952; Hallacher \& Robert 1985).

El IIR está determinado por la siguiente ecuación:

$$
I I R=(\% N+\% V) * \% F
$$

Donde $\% \mathrm{~N}$ y $\% \mathrm{~V}$ corresponden al porcentaje en número y volumen respectivamente de una presa en un estómago y \% F es la frecuencia de aparición de una presa en el conjunto de estómagos analizados. El volumen total y de cada tipo de presa, así como el volumen estomacal fue obtenido por desplazamiento en una probeta de $25 \mathrm{ml}$ (sensibilidad 0,2 $\mathrm{ml}$ ), según lo indicado por Hynes (1950).

Por su parte el ISP permite determinar la semejanza de los contenidos gástricos entre grupos, siendo expresado según la ecuación:

$$
I S P=100 \sum_{i=1}^{S} \min \left(a_{i}, b_{i}\right)
$$

Donde a y b son las proporciones en el índice de importancia relativa (IIR), de la i-ésima categoría de presa en la dieta de E. crassicaudus. S es el número total de categorías de presas comunes en la dieta. El rango de ISP varía entre 0 y $100 \%$, considerando significativo sobre el $50 \%$ de similaridad en la alimentación entre grupos (Hallacher \& Robert 1985). En el presente estudio se analizó el ISP entre meses, sexos y grupo de longitudes.
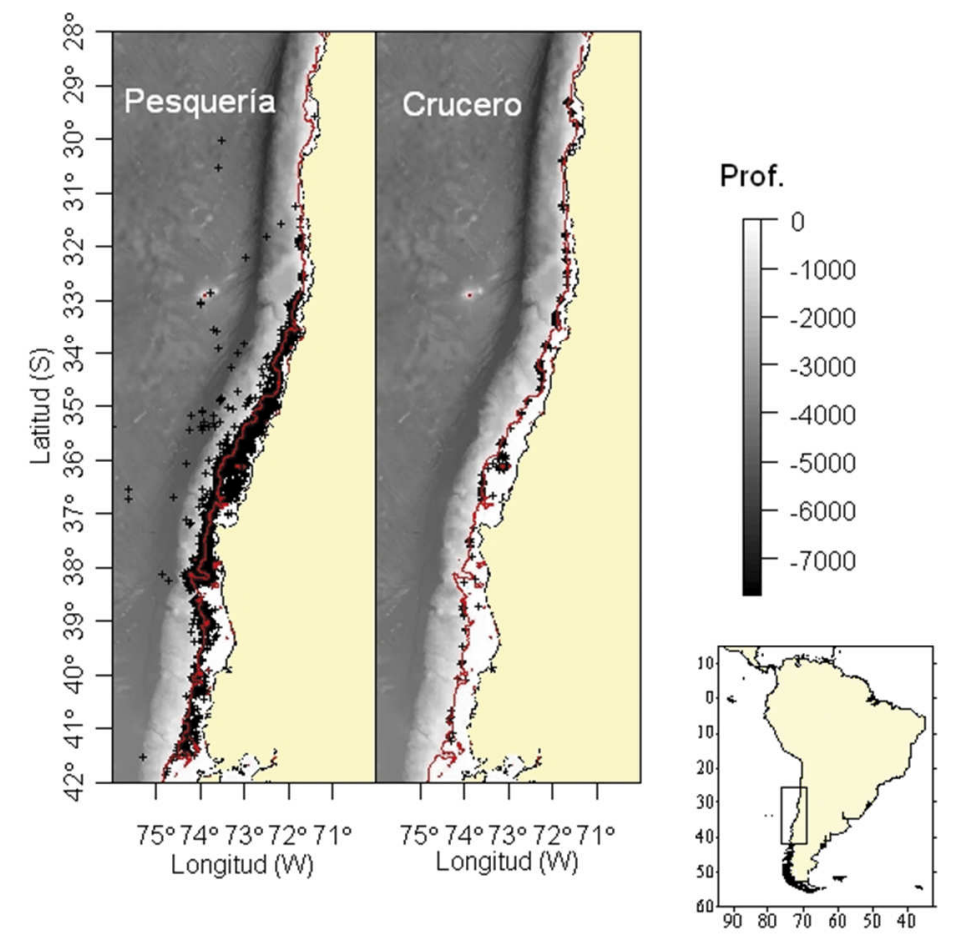

FiguRa 1. Distribución espacial de los lances de pesca de besugo. Desde la pesquería (periodo 1997-2005) y desde los lances positivos con besugo registrados en los cruceros de evaluación acústica de merluza común (periodo 1993-2005). Veril de los $500 \mathrm{~m}$ de profundidad (línea en rojo)

FIGURE 1. Spatial distribution of hauls of cardinal fish. From fishery (period 1997-2005) and from positive hauls with cardinal fish in the acoustic surveys of Chilean hake (period 1993-2005). Depth $500 \mathrm{~m}$ (red line). 


\section{RESULTADOS}

En el estudio de distribución y batimetría de $E$ crassicaudus, se analizó la información proveniente de 8016 lances realizados por la flota pesquera entre los años 1997 y 2006, concentrados entre Valparaíso (3330'S) y Valdivia (39 $50^{\circ}$ 'S). Por su parte, los lances con captura positiva de besugo en la prospección acústica de merluza común totalizaron 302 registros durante $\operatorname{los} 9$ años analizados. Se observaron capturas positivas de besugo en todo el rango de latitud que

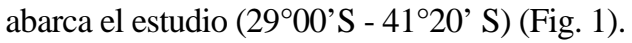

La frecuencia de lances realizados por la flota en relación a la latitud muestra una distribución relativamente uniforme a lo largo de su rango de operación, excepto al norte de los $34^{\circ} \mathrm{S}$ y al sur de $\operatorname{los} 38^{\circ} \mathrm{S}$, donde la frecuencia disminuye. Los lances positivos con presencia de besugo en la prospección anual de merluza común, coincidió con lo observado en la pesquería. Esta información además confirma una distribución más amplia de besugo hacia el norte con un importante número de lances positivos bajo $\operatorname{los} 34^{\circ} \mathrm{S}$ (Fig. 2).

\section{Pesquería}

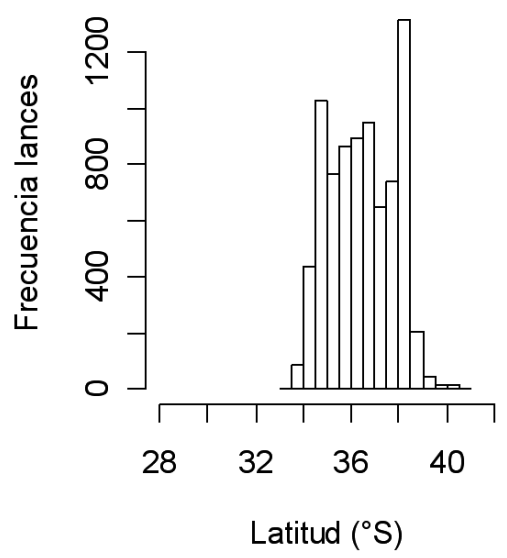

Crucero

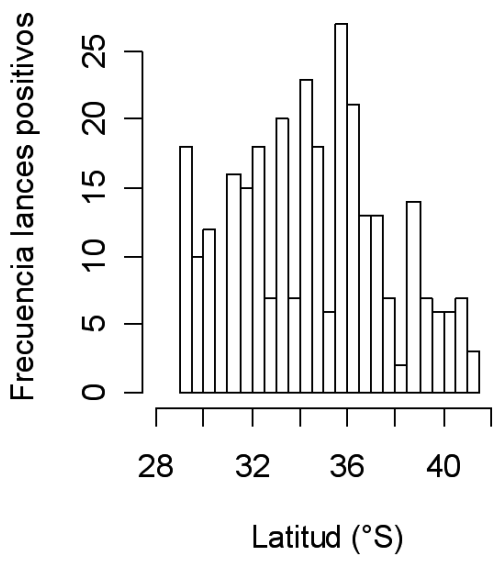

Figura 2. Distribución de frecuencia de lances de pesca realizados por la flota (1997-2005) y lances positivos con besugo en la evaluación acústica de merluza (1993-2005) en relación a la latitud.

FIGURE 2. Frequency distribution of fishing hauls made by the fleet (1997-2005) and cardinal fish hauls positive in the acoustic surveys of Chilean hake (1993-2005) in relation with latitude.

Las capturas mas altas de la flota se registraron alrededor de los $36^{\circ} \mathrm{S}$. Por su parte los lances de la prospección directa, registraron las mayores capturas entre los $34^{\circ} \mathrm{S}$ y $35^{\circ} \mathrm{S}$ (Fig. 3)

En cuanto a la batimetría, la flota concentró su operación sobre la plataforma y talud continental a profundidades de 130 y $600 \mathrm{~m}$, registrando la mayor frecuencia de lances entre los 350 y $400 \mathrm{~m}$. Este patrón coincide plenamente con los resultados del análisis de la información complementaria. De la misma forma el nivel de captura, mostró un patrón coincidente en ambas fuentes de información, obteniendo los mayores valores en el rango de profundidad entre 300 y $400 \mathrm{~m}$ (Fig. 4).

Respecto a la alimentación, las presas mas consumidas por E. crassicaudus en los meses analizados fueron organismos planctónicos representados por eufáusidos y estomatópodos. 
Distribución, batimetría y alimentación de besugo: LEAL, E. ET AL.

Aunque algunos componentes bentónicos de su dieta se identificaron en los meses de febrero y abril, su IIR fue bajo (Tabla I). De acuerdo al ISP, no se encontraron diferencias significativas en los hábitos alimenticios del besugo distribuido en la zona del golfo de Arauco en los meses estudiados. El ISP fue $82 \%, 75 \%$ y $74 \%$, entre los meses Enero-Febrero, Enero-Abril y Febrero-Abril respectivamente. De la misma forma este índice no evidenció diferencias en los hábitos alimenticios de machos y hembras $(94,5 \%)$ y entre grupos de longitudes $(94,9 \%)$.
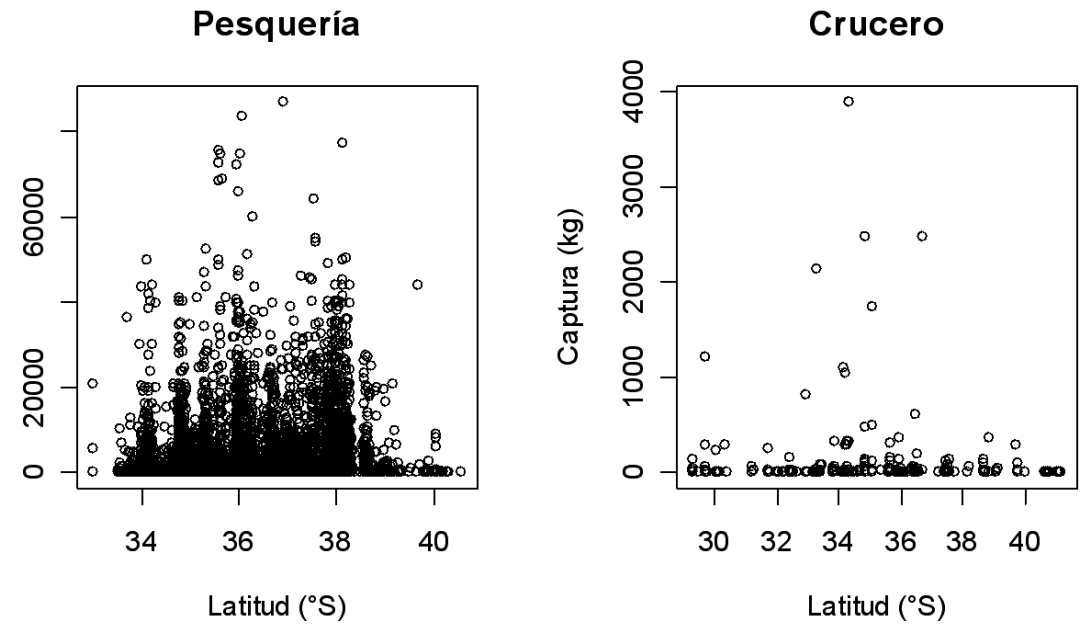

Figura 3. Capturas (kg) de besugo obtenidas por la flota (1997-2005) y en los cruceros de evaluación directa de merluza (1993-2005) en relación a la latitud.

FIGURE 3. Cardinal fish catch (kg) obtained by the fleet (1997-2005) and by acoustics surveys of Chilean hake (19932005) in relation with latitude.
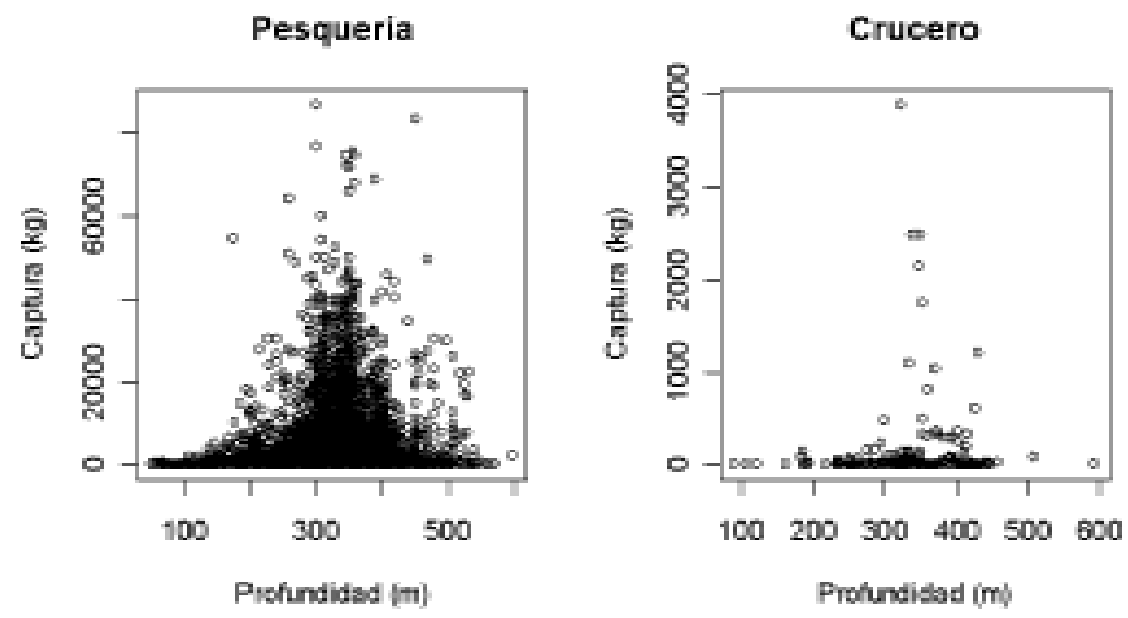

FiguRA 4. Capturas (kg) de besugo obtenidas por la flota (1997-2005) y en los cruceros de evaluación directa de merluza (1993-2005) en relación a la profundidad.

FIGURE 4. Cardinal fish catch (kg) obtained by the fleet (1997-2005) and by acoustics surveys of Chilean hake (1993-2005) in relation with depth. 
Gayana 73(1), 2009

TABLA 1. Valores de porcentajes en número, volumen, frecuencia e IIR para cada tipo de presa consumida por E. crassicaudus durante el mes de enero, febrero y abril.

TABLE 1. Percentage value in number, volume, frequency and IIR for each type of prey consumed by E. crassicaudus during January, February and April.

\begin{tabular}{|c|c|c|c|c|c|c|c|c|c|c|c|c|}
\hline \multirow[t]{2}{*}{ Item } & \multicolumn{4}{|c|}{ Enero $(\mathrm{n}=30)^{*}$} & \multicolumn{4}{|c|}{ Febrero $(n=25)^{*}$} & \multicolumn{4}{|c|}{ Abril $(n=46)^{*}$} \\
\hline & $\% \mathrm{~N}$ & $\% \mathrm{~V}$ & $\% \mathrm{~F}$ & FI.I.R & $\% \mathrm{~N}$ & $\% \mathrm{~V}$ & $\% \mathrm{~F}$ & \%I.I.R & $\% \mathrm{~N}$ & $\% \mathrm{~V}$ & $\% \mathrm{~F}$ & \%I.I.R \\
\hline Eufáusidos & 92 & 46 & 80 & 69 & 71 & 21 & 56 & 67 & 91 & 33 & 35 & 93 \\
\hline Stomatópoda & 1 & 3 & 13 & 61 & 17 & 11 & 20 & 7 & 0 & 1 & 4 & 0 \\
\hline Cephalópoda & $* *$ & $* *$ & $* *$ & $* *$ & 6 & 21 & 20 & 7 & $* *$ & $* *$ & $* *$ & $* *$ \\
\hline Caelorhynchus & $* *$ & $* *$ & $* *$ & $* *$ & 1 & 16 & 12 & 3 & $* *$ & $* *$ & $* *$ & $* *$ \\
\hline Heterocarpus reedi & $* *$ & $* *$ & $* *$ & $* *$ & 1 & 7 & 12 & 1 & 2 & 27 & 14 & 1 \\
\hline R.N.I. & 7 & 51 & 83 & 30 & 4 & 24 & 40 & 14 & 7 & 39 & 57 & 6 \\
\hline
\end{tabular}

* Estómagos con contenido

\section{DISCUSION}

Los resultados obtenidos en el presente estudio demostraron la presencia de besugo hasta el límite norte de los lances de pesca realizados en la evaluación directa de merluza común $\left(29^{\circ} \mathrm{S}\right)$. Esto permitió establecer una distribución más amplia del recurso de la reconocida a partir de la operación de la flota de pesca ( $\left.33^{\circ} 00^{\prime} \mathrm{S}\right)$. Aunque de acuerdo a antecedentes no documentados en literatura formal su distribución hacia el norte sería aún mas amplía, siendo reportada a partir de Taltal $\left(25^{\circ} 06^{\prime} \mathrm{S}\right)$.

En relación a la batimetría, la flota registra lances positivos con besugo a profundidades superiores a los $600 \mathrm{~m}$, aunque su operación se concentra sobre la plataforma y talud continental, obteniendo la mayor frecuencia de lances positivos y mayores capturas a profundidades entre 300 y $400 \mathrm{~m}$. Estos resultados coincidieron plenamente con los obtenidos a partir de la información independiente de la pesquería. Confirmando que esta especie estaría distribuida preferentemente dentro de ese rango de profundidades.

Respecto de sus hábitos tróficos, E. crassicaudus basó su alimentación principalmente en el consumo de especies pelágicas, cuyos representantes más relevantes fueron eufáusidos y estomatópodos. El resto de las presas no presentaron regularidad en su incidencia entre meses y según los valores de IIR no fueron significativas en la dieta de la especie en la zona y época estudiada. Sin embargo, la ocurrencia de un crustáceo asociado al fondo marino en el contenido estomacal de besugo revela su comportamiento como consumidor secundario relacionado al bentos. Y como consumidor terciario o de un orden superior al predar sobre peces y cefalópodos.

Aunque se aprecian ciertas diferencias en cuanto a la diversidad de especies, los resultados del presente estudio concuerdan con los obtenidos por Arancibia y Meléndez (1987), quienes otorgan a esta especie hábitos alimenticios pelágicos y epibentónicos. Por su parte Galvéz et al. (2000) reportan que la dieta de besugo en la zona central de Chile varia desde invertebrados (eufausidos) y pequeños peces pelágicos (mictófidos) hasta organismos típicamente bentónicos (langostinos, camarón, zapateador, pejerrata). De la misma forma Neira \& Arancibia (2004) sitúan a E. crassicaudus en un nivel trófico alto, indicando su impacto fundamentalmente sobre copépos eufausidos y fitoplancton. Otro representante del género (Epigonus elegans), en el 
Distribución, batimetría y alimentación de besugo: LEAL, E. ET AL.

Pácifico sureste es catalogado como un pez bentopelágico debido a las migraciones verticales diurnas entre el pélagos y el bentos (Abramov \& Lipskaya 1985).

De acuerdo a los antecedentes reportados para la especie y a los de resultados del presente estudio es posible sugerir, que determinado por la oferta ambiental de alimentos, E. crassicaudus presentaría hábitos migratorios en su distribución en la columna de agua. Distribuyéndose preferentemente sobre la plataforma y talud continental a profundidades de entre 300 y 400 m.

\section{AGRADECIMIENTOS}

Los autores agradecen al Instituto de Fomento Pesquero (IFOP). En particular al Departamento de Evaluaciones Directas y al personal del Programa de Investigación Situación Pesquería Demersal Centro Sur y Aguas Profundas por la disponibilidad de la información para llevar a cabo este estudio.

\section{BIBLIOGRAFIA}

Abramov, A. 1992. Species Composition and Distribution of Epigonus (Epigonidae) in the Word Ocean. Journal of Ichthyology. 32(5), 94-108.

Abramov, A. \& Lipskaya, H. 1989. On the feeding of Epigonus elegans in the Region of the Nazka Ridge. Voprosy ikhtiologii/Journal of Ichthyology. 29(1): 129-134.

Arancibia, H. \& Meléndez, R. 1987. Alimentación de peces concurrentes en la pesquería de Pleuroncodes monodon Milnes Edwards. Investigaciones Pesqueras. 34: 113-129.

BERG, J. 1979. Discussion of methods of investigating the food of fish, with reference to a preliminary study of the prey of Gobiusculus flavescens (Gobiidae). Marine Biology. 50: 263-273.

Gálvez, M., Rebolledo, H., Pino, C., Cubillos, L., Sepúlveda, A. \&. Rojas, A. 2002. Parámetros biológico-pesqueros y evaluación de stock de besugo (Epigonus crassicaudus). Resporte Técnico. Instituto de Investigación Pesquera. Informe Final, 110 pp.

Hallacher, L. \&. ROBERT, D. 1985. Differential utilization of space and food by inshore rockfishes (Scorpaenidae: Sebastes) of Carmel Bay, California. Environmental Biology of Fishes. 12:91-110.

Hynes, H. 1950. The food of freshwater sticklebacks (Gasterosteus aculeatus and Pygosteus pungitius) in study of the food fishes. Journal of Animal Ecology. 19: 36-58.

Johnson, G.D. 1984. Percoidei: Development and relationship. In: Ontogenics and systematics of Fishes. American Society of Ichthyologists and Herpetologists. 1: 464-498.

Lillo, S., Olivares, J., Braun, M., Nuñez, S., Saavedra, A., SaAvedra, J. \& Molina, E. 2006. Evaluaciones Hidroacústicas de merluza común año 2005. Fondo de Investigación Pesquera (FIP 2005-05). Informe Final. 238 pp.

Neira, S. \& Arancibia H. 2004. Comparative analysis of trophic structure of commercial fishery species off Central Chile in 1992 and 1998. Ecological Modelling 172:233-248.

PARIN, N. 1987. The system of oceanic. Ichthyocence resursy otkrytogo okeana (Biological resources of the open ocean). Nauka, Moscow, pp. 138-163.

PinKas, L., Oliphant, M. \& Iverson, I. 1971. Food habits of albacore, bluefin tuna, and bonito in California waters. Fisheries Bulletin.152: 1-105.

Servicio Nacional de Pesca. 1992. Anuario Estadístico de Pesca Chile. (http://www.sernapesca.cl). Ministerio de Economía, Fomento y Reconstrucción, Santiago, Chile.

Wiff, R., Quiroz, JC. \& TAscheri, R. 2005. Estado de explotación del recurso besugo (Epigonus crassicaudus) en Chile. Investigaciones Marinas. 33 (1): 57-67.

WifF, R., Quiroz, JC., TASChERI, R \& CONTRERAS, F. 2008. Effect of fishing tactics on the standardization of cardinalfish (Epigonus crassicaudus) catch rates in the demersal multispecies fishery off central Chile. Ciencias Marinas. 34(2): 143-154.

WhitTAKER, R.H. 1952. A study of summer foliage insect community in the Great Smokey Mountains. Ecological Monographs. 22:1-44. 